\title{
Mechanical Composition Control for Ti-Based Hydrogen Storage Alloys*
}

\author{
Saule Zholdayakova ${ }^{\dagger}$ \\ Department of Science and Technology, Tokai University, Kitakaname 4-1-1, Hiratsuka 259-1292, Japan \\ Ryota Gemma \\ Department of Materials Science, Tokai University, Kitakaname 4-1-1, Hiratsuka 259-1292, Japan \\ Haru-Hisa Uchida \\ Department of Human Development, Tokai University, Kitakaname 4-1-1, Hiratsuka 259-1292, Japan \\ Masashi Sato \\ Department of Applied Chemistry, Tokai University, Kitakaname 4-1-1, Hiratsuka 259-1292, Japan \\ Yoshihito Matsumura \\ Department of Nuclear Engineering, Tokai University, Kitakaname 4-1-1, Hiratsuka 259-1292, Japan
}

(Received 26 January 2018; Accepted 24 April 2018; Published 21 June 2018)

\begin{abstract}
Hydrogen absorbing alloys have attracted great attention because they are a safe and efficient media for transporting hydrogen energy. $\mathrm{AB}, \mathrm{AB}_{2}, \mathrm{AB}_{5}$, and $\mathrm{A}_{2} \mathrm{~B}$-type hydrogen storage compounds and related substituted multi-component alloys have been proposed. In general, titanium-based alloys are among the most promising materials for hydrogen storage. Among the various available types of metal hydrides, $\mathrm{AB}_{2}$ type Ti-Cr-based alloys are the most promising candidates. In particular, there have been many methods for improving the hydrogen sorption properties. One of them is to prepare hydrogen absorbing alloys by mechanical alloying (MA). MA also enables synthesizing various non-equilibrium alloys and has been widely applied to modify properties of hydrogen storage alloys. Thus, MA is possibly the best way for mechanical composition control and mass production of various hydrogen storage alloys. [DOI: 10.1380/ejssnt.2018.298]
\end{abstract}

Keywords: Energy technology; Chromium; Manganese; Titanium; Alloys; Powders; Hydrides

\section{INTRODUCTION}

With the growing cost of energy and the seriousness of global warming, it has become vitally important to find sources of clean and sustainable energy. Hydrogen has attracted a great deal of consideration in recent years as a feasibly ideal energy carrier. Its application for mobility and portable electronics is to a large extent limited by the difficulty of finding an available storage method [1]. Developing of hydrogen storage compounds with high structural stability and hydrogen storage capacity makes the practical usage of hydrogen realizable [2]. Hydrogen absorbing alloys have attracted great attention because they are a safe and efficient media for transporting hydrogen energy [3].

Multicomponent alloys of hydrogen storage compounds ( $\mathrm{AB}, \mathrm{AB}_{2}, \mathrm{AB}_{5}$, and $\mathrm{A}_{2} \mathrm{~B}$-type) have been broadly studied in the last 3-4 decades. Subsequently, the electrodes of $\mathrm{AB}_{5}\left(\mathrm{LaNi}_{5}\right)$ - and $\mathrm{AB}_{2}\left(\mathrm{ZrV}_{2}\right)$-type alloys have already been implemented in rechargeable nickel-metal hydride $(\mathrm{Ni} / \mathrm{MH})$ batteries. The representative alloys among the most promising materials for hydrogen storage are titanium-based alloys, because of their storage property and cost-effectiveness [1].

In general, Ti based alloys have been the Laves phase $\mathrm{TiMn}_{2}$ based one [3]. The hydrogen capacity of the Tibased Laves phase alloy limited 2 mass\%. Body centered

\footnotetext{
* This paper was presented at the 8th International Symposium on Surface Science, Tsukuba International Congress Center, Tsukuba, Japan, October 22-26, 2017.

† Corresponding author: saulezholdayakova@yahoo.com
}

cubic (BCC) metals and alloys intrinsically have a large hydrogen capacity. Ti-based BCC solid solution alloys are both single and multiphase alloys. They were investigated and developed from a new concept "Laves phase related BCC solid solution" [3]. They showed a hydrogen capacity of 2.2 mass\%. Ti-based $\mathrm{AB}_{2}$ alloys consisted of $\mathrm{BCC}$ and Laves phases and every phase contributed to hydrogenation. In particular, the BCC phase absorbed hydrogen in a favorable manner like the intermetallic compounds and showed the same hydrogenation pressure (equilibrium pressure) as other component phases. The BCC solid solution phase that appears with the Laves phases is a promising candidate for a hydrogen absorbing alloy [3].

Among the various available types of metal hydrides, $\mathrm{AB}_{2}$ type Ti-Cr-based alloys are the most promising candidates due to their relatively high desorption pressure plateaus and moderate hydrogen storage capacities, fast kinetics, and easy activation. However, they are still far away from the targets for high pressure alloys for use in hybrid systems due to their relatively high hydrogen desorption pressures and low capacities. For example, $\mathrm{TiCr}_{2}$ has an extreme high absorption plateau of $100 \mathrm{MPa}$ even at a low temperature of $195 \mathrm{~K}$, which is much higher than the maximum working pressure of a hybrid tank. Alloying with other elements is one of the most effective ways to adjust the formation enthalpy and cell volume of metal hydrides, which allows considerable influence over the hydrogen absorption/desorption pressure and capacity [4]. The ternary intermetallic TiCrMn is partially manganese substituted derivative of the $\mathrm{C} 14$ Laves phase $\mathrm{TiCr}_{2}$.

Because of the outstanding physical and chemical properties, the C14 Laves phase TiCrMn has attracted a lot of attention, especially for its potential application as a hy- 
TABLE I. Result of EDS analysis of samples milled by dry type.

\begin{tabular}{ccc}
\hline \hline \multirow{2}{*}{ Element } & $\begin{array}{c}\text { MA-treated in stainless steel pot } \\
\text { Atom percent (at\%) }\end{array}$ & $\begin{array}{c}\text { MA-treated in ceramic pot } \\
\text { Atom percent (at\%) }\end{array}$ \\
\hline $\mathrm{Ti}$ & 49.11 & 14.34 \\
$\mathrm{Cr}$ & 40.12 & 6.25 \\
$\mathrm{Mn}$ & 10.77 & 29.38 \\
$\mathrm{Al}$ & & 13.75 \\
$\mathrm{O}$ & & 36.27 \\
\hline \hline
\end{tabular}

drogen storage material. Due to this partial substitution of the Cr by Mn, the properties of host (hydrogen-free) intermetallic and its corresponding hydride phases are also altered [2].

A more suitable manufacturing method for mass production of alloys is to apply mechanical milling by using a planetary ball mill. Mechanical alloying (MA) is able to synthesize various non-equilibrium alloys so that it has been widely applied to modify properties of a hydrogen storage alloy [5]. It is well known that the TiFe alloy also synthesized by MA has been extensively studied [1, 6-9]. The aim of the present research is to study mechanical composition control for a $\mathrm{Ti}$ based hydrogen storage alloy and discuss about mass production of TiCrMn as a hydrogen storage alloy as a model hydrogen carrier. For example, in Kazakhstan, Ti, Cr, and Mn are abundant and their prices are less expensive [10]. Therefore, the future potential of renewable energy is high $[11,12]$. Hydrogen could be a conductor of energy to increase utilization of renewable energy there. As a very preliminary study, we first prepared TiCrMn alloys in wet and dry ball-milling conditions, respectively.

\section{EXPERIMENTAL}

Ti, Cr, and Mn powders of $99 \%$ purity were mixed with an atomic ratio of $1: 1: 1$. For the MA in dry condition, a mixture of the powder was sealed with stainless steel balls in a stainless steel pot. Alternatively, ceramic balls and a ceramic pot were also employed to compare the results with stainless steel balls and pot. In both MA treatments, MA was rottated at a speed of $350 \mathrm{rpm}$ for $50 \mathrm{~h}$ by planetary ball-mill (Retsch, Planetary Ball Mill PM 100) with the ball to sample ratio in weight of $1: 10$, using milling balls with a diameter of $8 \mathrm{~mm}$.

For the MA treatment in wet condition, the pot was filled with hexane (super dehydrated) as a dispersion medium up to $2 / 3$ of the volume of the pot made of stainless steel and ball-milled using stainless steel balls. Different milling times $(10,20,50$, and $100 \mathrm{~h})$ were applied so as to produce powders with different morphology and microstructure.

The change in particle size of the powders was examined by scanning electron microscopy (SEM, JCM-6000Plus NeoScope $\left.{ }^{\mathrm{TM}}\right)$. The compositional analysis of the alloys was carried out by Energy Dispersive X-ray spectroscopy (EDX). The phase identification of all of the prepared samples was carried out by X-ray diffraction (XRD) using Rigaku Mini Flex 600.

\section{RESULTS AND DISCUSSIONS}

\section{A. MA in dry condition}

The results of the EDX analysis on powders obtained by dry MA condition using stainless steel and ceramic pots are shown in Table I. In case of the dry MA treatment in a stainless steel pot, $\mathrm{Mn}$ concentration in the sample powders after the MA showed much lower concentration than expected. This was caused by significant fixation of Mn on the inner wall of the pot, as confirmed by eyesight after the MA treatment. The same MA treatment using a ceramic pot also resulted in fixation and caused large subtraction of the composition from the nominal composition of $\mathrm{Ti}$ : $\mathrm{Cr}: \mathrm{Mn}=1: 1: 1$, as shown in Table I. In addition, significant amounts of $\mathrm{Al}$ and $\mathrm{O}$ were detected, reaching 16.22 and 10.37 at\%, respectively. This contamination of the sample powders by $\mathrm{Al}$ and $\mathrm{O}$ originates from the ceramic pot and balls, which means fine particles of $\mathrm{Al}_{x} \mathrm{O}_{y}$ were mixed with powders of $\mathrm{Ti}, \mathrm{Cr}$, and $\mathrm{Mn}$.

The results suggested that the fixation cannot be avoided unless a dispersion medium is used for the MA. In the next section, the results of the MA in wet condition are shown.

\section{B. MA in wet condition}

The particle size of milled powders decreased with increasing MA time, while the morphology did not show a clear difference as shown in Fig. 1. For each powder sample, EDX analysis was carried out at different region of the SEM image in order to confirm compositional homogeneity. The respective zones, a, b, and c are marked in each image shown in Fig. 1.

The results of EDX are shown in Table II. Ti, Cr, and $\mathrm{Mn}$ powers were mixed to the target amount in an atomic ratio of $1: 1: 1$. Milling for $10 \mathrm{~h}$ and $20 \mathrm{~h}$ resulted in the composition ratio of $\mathrm{Ti}: \mathrm{Cr}: \mathrm{Mn}=1.00: 2.61: 1.50$ (zone a) and $1.00: 0.69: 0.54$ (zone a), respectively. The composition of the samples was not identical to the target values in an atomic rationof $1: 1: 1$. At a prolonged milling for $50 \mathrm{~h}$, the composition showed $1.00: 1.02: 1.19$ (zone a), which is close to the nominal composition. However, regarding the results of $\mathrm{b}$ and $\mathrm{c}$ zones focused in grains, the composition was different, suggesting that the MA is still insufficient. MA treatment for $100 \mathrm{~h}$, the longest milling time in this study, marked the composi- 

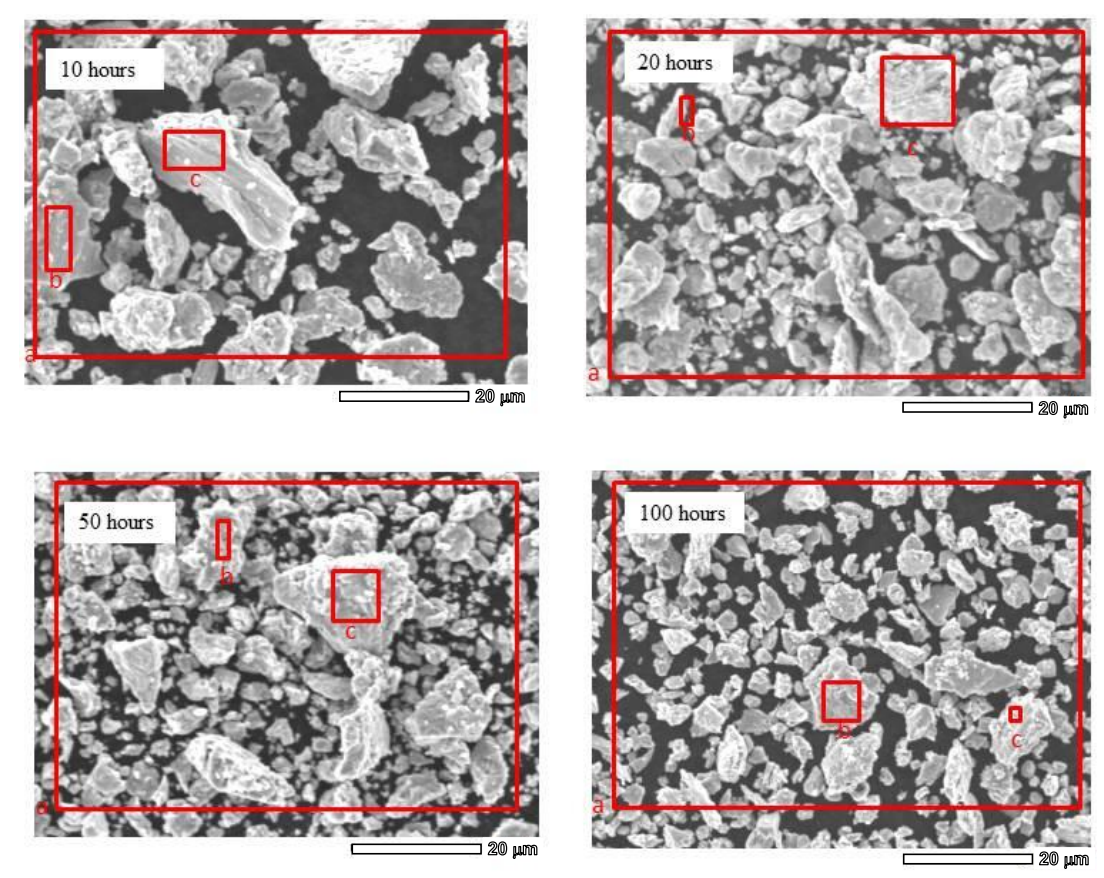

FIG. 1. SEM images show a different particle size at different milling time and region of EDX analysis with a, b, c zones.

TABLE II. Result of EDS analysis of samples milled by wet type for different period.

\begin{tabular}{cccccc}
\hline \hline \multicolumn{2}{c}{ Time } & $10 \mathrm{~h}$ & $20 \mathrm{~h}$ & $50 \mathrm{~h}$ & $100 \mathrm{~h}$ \\
\hline Zone & Element & Atomic percent (at\%) & Atomic percent (at\%) & Atomic percent (at\%) & Atomic percent (at\%) \\
\hline \multirow{2}{*}{$\mathrm{a}$} & $\mathrm{Ti}$ & 48.81 & 23.34 & 35.53 & 32.19 \\
& $\mathrm{Cr}$ & 18.70 & 33.64 & 34.68 & 32.61 \\
& $\mathrm{Mn}$ & 32.49 & 43.03 & 29.79 & 35.19 \\
\hline & $\mathrm{Ti}$ & 50.70 & 16.66 & 37.94 & 31.28 \\
$\mathrm{~b}$ & $\mathrm{Cr}$ & 16.19 & 66.67 & 30.05 & 36.86 \\
& $\mathrm{Mn}$ & 33.11 & 16.67 & 32.01 & 31.86 \\
\hline & $\mathrm{Ti}$ & 50.14 & 21.28 & 30.61 & 32.19 \\
$\mathrm{c}$ & $\mathrm{Cr}$ & 19.96 & 46.86 & 27.15 & 29.74 \\
& $\mathrm{Mn}$ & 29.90 & 31.86 & 42.24 & 38.07 \\
\hline \hline
\end{tabular}

tion of $1.00: 1.01: 1.07$ (zone a) and $1.00: 1.17: 1.01$ in particles, indicating that the compositional difference between average scale and individual particle decreased as the milling time increased. Even longer MA treatment could possibly completely homogenize the composition.

Figure 2 shows the XRD patterns of the TiCrMn mixtures mechanically alloyed with different milling times. After a milling time of $10 \mathrm{~h}$, the peaks of the TiCrMn phase were found. But, Ti, Cr, and Mn peaks are still present. In particular, a strong Ti peak of $2 \theta=40.2^{\circ}$ was evident. Milling for $20 \mathrm{~h}$ lead to a reduction of the Ti peak. After $50 \mathrm{~h}$ of milling, the XRD pattern was dominated by peaks from the TiCrMn phase. In fact, the height of the $\mathrm{Ti}, \mathrm{Cr}$, and $\mathrm{Mn}$ peaks became the background level. After the MA was milled for $100 \mathrm{~h}$, the XRD result suggests almost a single phase of TiCrMn. This is consistent with the EDX results, which focused on a mechanical composition control for mass production of hydrogen storage alloys. Using the process of ball milling by wet type with different times obtained different morphology and microstructure. The evolution of the particle size was determined. The alloy obtained from $100 \mathrm{~h}$ of milling had the smallest average particle size and the closest composition to the target atomic ratio of elements.

\section{CONCLUSIONS}

In this research, Ti-based $\mathrm{AB}_{2}$ alloys were prepared solely by MA using a ball-milling process. We have obtained TiCrMn alloys from a mixture of $\mathrm{Ti}, \mathrm{Cr}$, and $\mathrm{Mn}$ using different ball milling times with hexane. It was shown that a dispersion medium like hexane is necessary to avoid fixation of the powders in the milling pot. By using this wet type ball milling process, the composition of the alloys could be controlled. We have successfully achieved a target with a composition with an atomic ratio of $1: 1: 1$ after $100 \mathrm{~h}$ of milling. This method has 


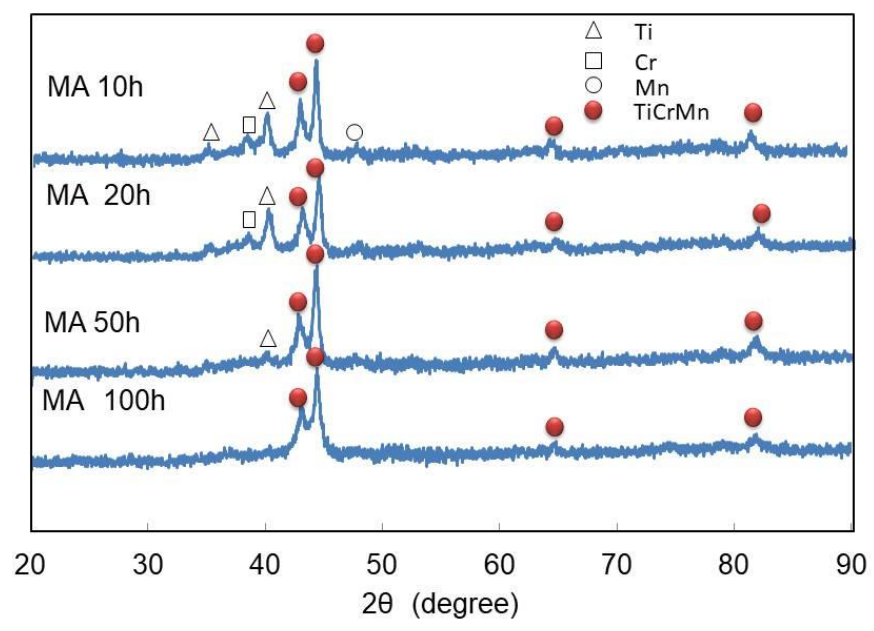

FIG. 2. X-ray patterns of the TiCrMn powders milled for different times. the potential for mass production of TiCrMn and offers a chance to utilize hydrogen storage alloys as a model of a hydrogen carrier. Moreover, chemical composition, crystal structure, and grain size might be controlled by changing the milling time in order to fine-tune the hydrogen storage property.

\section{ACKNOWLEDGMENTS}

We would like to express our sincere gratitude to the Tokai University Imaging Center for Advanced Research, the College Women's Association of Japan, Isehara Rotary Club Japan, and the "Bolashak" Presidential Scholarship Program of Republic Kazakhstan.
[1] B. Abrashev, T. Spassov, M. Pandev, S. Vassilev, and A. Popov, Belg. Chem. Commun. 49, 247 (2017).

[2] Z.-S. Nong, J.-C. Zhu, X.-W. Yang, Y. Cao, Z.-H. Lai, Y. Liu, and W. Sun, Solid State Sci. 32, 1 (2014).

[3] E. Akiba, Curr. Opin. Solid State Mater. Sci. 4, 267 (1999).

[4] Z. Cao, L. Ouyang, H. Wany, J. Liu, D. Sun, Q. Zhang, and M. Zhu, Int. J. Hydrogen Energy 40, 2717 (2015).

[5] M. Abe and T. Kuji, J. Alloys Compd. 446-447, 200 (2007).

[6] T. Ikeuchi, T. Saito, and M. Kobayashi, J. Jpn. Soc. Powder Powder Metall. 49, 861 (2002).
[7] H. Aoyagi, K. Aoki, and T. Matsumoto, J. Alloys Compd. 231, 804 (1995).

[8] H. Hotta, M. Abe, T. Kuji, and H. Uchida, J. Alloys Compd. 439, 221 (2007).

[9] H. Miyamura, J. Jpn. Inst. Light Metals 55, 629 (2005).

[10] http://www.stat.gov.kz/ Statistic Agency of Kazakhstan.

[11] M. Karatayev and M. L. Clarke, Energy Procedia 59, 97 (2014).

[12] M. Karatayev and M. L. Clarke, Renew. Sustain. Energy Rev. 55, 491 (2016). 